\section{Association of Human Leukocyte Antigen and Cytomegalovirus disease after Kidney Transplantation}

DOI: 10.1515/rrlm-2017-0035

\section{Dear Editor,}

Cytomegalovirus (CMV) is the most important infectious cause of morbidity and mortality in transplantation. More and more patients are transplanted and because of the increasing immune-modulating agents, the risk for developing CMV disease in those patients is increasing. CMV is a DNA virus from human herpesviruses class. Human cytomegalovirus-human herpesvirus 5 belongs to order Herpesvirales, family Herpesviridae, subfamily Betaherpesvirinae, genus Cytomegalovirus, species Human herpesvirus [1]. There are three important mechanisms of CMV infection acquisition in patients with solid organ transplants [2,3]: primary infection (D+/R-), reactivation infection (D-/R+) and superinfection $(\mathrm{D}+/ \mathrm{R}+)$. a) Primary infection with CMV occurs in case of transplantation of a CMV negative recipient (R-) from a positive donor $(\mathrm{D}+)$. This $\mathrm{D}+/ \mathrm{R}-$ serologic mismatch is estimated to occur in $15 \%$ to $25 \%$ of all solid organ transplantation [4]. In the absence of antiviral prophylaxis, a $\mathrm{D}+/ \mathrm{R}$ - serologic mismatch will almost always result in the transmission of CMV to the susceptible transplant recipient where it can cause clinically severe primary CMV disease. Primary CMV infection may occur in R- when $\mathrm{CMV}$ is transmitted through blood transfusion from $\mathrm{D}+$ or through natural transmission routes in community [5]; b) The CMV infection can reactivates in an CMV positive recipient during the periods of decreased immunity after kidney/ pancreas transplantation from a CMV negative donor $(\mathrm{D}-/ \mathrm{R}+)$. The degree of CMV reactivation and replication in $\mathrm{R}+$ patients is relatively lower in comparison with the primary CMV infection in $\mathrm{D}+/ \mathrm{R}$ - patients because patients have preexisting CMV specific cell-mediated and humoral immunity, so they have a relatively lower risk of developing symptomatic CMV disease [6]. c) Superinfection/reinfection occurs when an CMV positive recipient is infected from a positive donor (or other exogenous source). In this way, the exogenous CMV transmitted through allograft and reactivated endogenous CMV can cause clinical disease after kidney/pancreas transplantation [6]. The predominant virus that reactivates in $\mathrm{CMV} \mathrm{D}+/ \mathrm{R}+$ patients is, in the majority of cases, donor-derived CMV [7,8] suggesting a potentially incomplete degree of cross-protection against other viral strains. There are important differences between "CMV infection" and "CMV invasive disease":

a. CMV infection assumes detection of CMV pp65 antigen in blood leukocytes in the absence of clinical manifestations or organ function abnormalities.

b. CMV disease was defined as the association of documented CMV infection with clinical symptoms, such as unexplained fever and leucopenia combined with the presence of the virus and/or histopathologic or immunohistochemical diagnosis of CMV in tissue samples.

In immunosuppressed seropositive patients, the presence of CMV into urine is common in the absence of invasive disease. The CMV disease diagnosis should be made only in the presence of microbiologic data, even if clinically would be important to initiate therapy on the moment of identification of CMV in urine [9]. 
The HLA system is known to play an important role in susceptibility and resistance to many infectious diseases. The HLA system plays a significant role in acceptance or rejection of a transplant $[10,11]$. Polymorphisms of the HLA will impact susceptibility to CMV infection, disease progression and treatment. The influence of HLA allele on CMV disease may be protective, predisposing or neutral. The impact of HLA on CMV disease is examined by analysis of allelic influences for each HLA-A, HLA-B, and HLADRB1. The relationship of each allele to CMV disease is expressed through an OR calculated of all alleles at each locus. We have analysed the association of particular HLA alleles and CMV disease after transplantation. This study included 674 kidney transplantations between January 2009 and December 2014 in Clinical Institute of Urology and Renal Transplantation Cluj-Napoca. The patients were divided into two groups according to the presence or absence of CMV disease. All recipients were positive CMV IgG and negative CMV IgM.
We have identified the HLA alleles association of 639 transplanted patients without CMV disease and 35 renal transplanted patients with CMV disease. HLA-A, HLA-B typing was performed by the standard microlymphocytotoxicity method.HLA-DR antigens were determined by the DNA-based PCR-SSP and PCR-SSO techniques. We have used innuPREP Blood DNA Mini kit (Analytik Jena) to extract DNA from whole blood. We have tested CMV antigenemia in circulating peripheral blood leukocytes using immunofluorescence assay for detection of CMV pp65 antigen (Merck). In our center, we use the prophylaxis for CMV disease in all kidney transplants. All the transplanted patients are receiving oral Valganciclovir for 3 months. We perform weekly monitoring of CMV by pp65 antigenemia in patients with CMV disease. In case of serious CMV disease (including most patients with tissue invasion), all patients are treated with intravenous Ganciclovir. The significance of difference between variables was assessed by Fischer's exact test. P values less than 0.05 were considered significant. Odds Ratio (OR) were

Table 1a. HLA - A alleles and CMV disease in kidney transplantation patients

\begin{tabular}{|c|c|c|c|c|}
\hline Allele HLA-A & $\begin{array}{c}\text { Alleles from patients with } \\
\text { CMV disease } \\
70 \\
\end{array}$ & $\begin{array}{c}\text { Alleles from patients without CMV } \\
\text { disease } \\
1278 \\
\end{array}$ & OR & p value \\
\hline 1 & $4(5.71 \%)$ & $182(14.24 \%)$ & 0.365 & 0.7126 \\
\hline 2 & $27(38.57 \%)$ & $389(30.43 \%)$ & 1.435 & 0.9816 \\
\hline 3 & $7(10 \%)$ & $120(9.39 \%)$ & 1.072 & 0.9479 \\
\hline 11 & $2(2.85 \%)$ & $96(7.51 \%)$ & 0.362 & 0.7052 \\
\hline $23(9)$ & $3(4.28 \%)$ & $38(2.97 \%)$ & 1.461 & 0.9221 \\
\hline $24(9)$ & $10(14.28 \%)$ & $164(12.82 \%)$ & 1.140 & 0.9673 \\
\hline $25(10)$ & $1(1.42 \%)$ & $46(3.59 \%)$ & 0.460 & 0.7361 \\
\hline $26(10)$ & $3(4.28 \%)$ & $56(4.38 \%)$ & 0.977 & 0.9172 \\
\hline $29(19)$ & $1(1.42 \%)$ & $13(1.01 \%)$ & 1.410 & 0.9346 \\
\hline $30(19)$ & 0 & $10(0.78 \%)$ & 0 & 0.5748 \\
\hline $31(19)$ & $4(5.71 \%)$ & $46(3.6 \%)$ & 1.623 & 0.8733 \\
\hline $32(19)$ & $2(2.85 \%)$ & $51(3.99 \%)$ & 0.707 & 0.8241 \\
\hline $33(19)$ & $3(4.28 \%)$ & $19(1.48 \%)$ & 2.967 & 0.5061 \\
\hline $34(10)$ & 0 & $1(0.07 \%)$ & 0 & 0.5748 \\
\hline 36 & $1(1.42 \%)$ & $1(0.07 \%)$ & 18.507 & 0.0008 \\
\hline $66(10)$ & 0 & $8(0.62 \%)$ & 0 & 0.5748 \\
\hline $68(28)$ & $2(2.85 \%)$ & $36(2.81 \%)$ & 1.014 & 0.9300 \\
\hline $69(28)$ & 0 & $2(0.15 \%)$ & 0 & 0.5748 \\
\hline
\end{tabular}


calculated and used to appreciate the association of CMV disease and HLA alleles in the transplanted patients. $\mathrm{OR}<1$ means protective allele; $\mathrm{OR}>1$ means predisposing allele; $\mathrm{OR}=1$, neutral allele.

From a total of 674 patients transplanted in CIUTR Cluj Napoca between 2009 and 2014, 416 patients were males and 258 patients were females. The age of patients was 4-74 years.
Thirty-five recipients $(5.2 \%)$ with positive CMV pp65 antigenemia developed CMV disease.

The results of HLA typing in patients with/ without CMV disease are presented in Tables $1 \mathrm{a}, 1 \mathrm{~b}$ and $1 \mathrm{c}$. Relation of each allele to CMV disease was expressed through an OR calculated in the context of all allele at each locus. The HLA alleles frequencies were determined in patients with CMV disease and recipients without

Table 1b. HLA - B alleles and CMV disease in kidney transplantation patients

\begin{tabular}{|c|c|c|c|c|}
\hline $\begin{array}{c}\text { Allele } \\
\text { HLA-B }\end{array}$ & $\begin{array}{c}\text { Alleles from patients with } \\
\text { CMV disease } \\
70 \\
\end{array}$ & $\begin{array}{c}\text { Alleles from patients with- } \\
\text { out CMV disease } \\
1249 \\
\end{array}$ & OR & $p$ value \\
\hline 7 & $5(7.14 \%)$ & $73(5.84 \%)$ & 1.269 & 0.7776 \\
\hline 8 & $3(4.28 \%)$ & $108(8.65 \%)$ & 0.485 & 0.7184 \\
\hline 13 & $2(2.85 \%)$ & $47(3.76 \%)$ & 0.770 & 0.9032 \\
\hline 17 & 0 & $2(0.16 \%)$ & 0 & 0.4086 \\
\hline 18 & $8(11.42 \%)$ & $145(11.6 \%)$ & 1.008 & 0.9382 \\
\hline 27 & $5(7.14 \%)$ & $72(5.76 \%)$ & 1.288 & 0.7666 \\
\hline 35 & $7(10 \%)$ & $195(15.61 \%)$ & 0.617 & 0.8193 \\
\hline 37 & 0 & $14(1.12 \%)$ & 0 & 0.4086 \\
\hline $38(16)$ & $4(5.71 \%)$ & $49(3.92 \%)$ & 1.520 & 0.6318 \\
\hline $39(16)$ & $1(1.42 \%)$ & $25(2 \%)$ & 0.726 & 0.8710 \\
\hline 40 & $1(1.42 \%)$ & $17(1.36 \%)$ & 1.075 & 0.8932 \\
\hline 41 & $1(1.42 \%)$ & $27(2.16 \%)$ & 0.671 & 0.8336 \\
\hline 42 & 0 & $1(0.08 \%)$ & 0 & 0.4086 \\
\hline $44(12)$ & $10(14.28 \%)$ & $112(8.97 \%)$ & 1.735 & 0.5544 \\
\hline $45(12)$ & 0 & $4(0.32 \%)$ & 0 & 0.4086 \\
\hline 46 & 0 & $2(0.16 \%)$ & 0 & 0.4086 \\
\hline 47 & $1(1.42 \%)$ & $9(0.72 \%)$ & 2.043 & 0.3677 \\
\hline 48 & $1(1.42 \%)$ & $3(0.24 \%)$ & 6.159 & 0.0011 \\
\hline $49(21)$ & $1(1.42 \%)$ & $33(2.64 \%)$ & 0.546 & 0.7495 \\
\hline $50(21)$ & $1(1.42 \%)$ & $14(1.12 \%)$ & 1.308 & 0.7438 \\
\hline $51(5)$ & $8(11.42 \%)$ & $119(9.52 \%)$ & 1.256 & 0.7924 \\
\hline $52(5)$ & 0 & $18(1.44 \%)$ & 0 & 0.4086 \\
\hline 53 & $1(1.42 \%)$ & $7(0.56 \%)$ & 2.631 & 0.1854 \\
\hline $55(22)$ & $1(1.42 \%)$ & $18(1.44 \%)$ & 1.014 & 0.9335 \\
\hline $56(22)$ & 0 & $11(0.88 \%)$ & 0 & 0.4086 \\
\hline $57(17)$ & $3(4.28 \%)$ & $12(0.96 \%)$ & 4.723 & 0.0113 \\
\hline $58(17)$ & $1(1.42 \%)$ & $10(0.8 \%)$ & 1.837 & 0.4562 \\
\hline $60(40)$ & $1(1.42 \%)$ & $14(1.12 \%)$ & 1.308 & 0.7438 \\
\hline $61(40)$ & $1(1.42 \%)$ & $20(1.6 \%)$ & 0.911 & 0.9972 \\
\hline $62(15)$ & 0 & $8(0.64 \%)$ & 0 & 0.4086 \\
\hline $63(15)$ & $1(1.42 \%)$ & $7(0.56 \%)$ & 2.631 & 0.1854 \\
\hline $64(14)$ & $2(2.85 \%)$ & $14(1.12 \%)$ & 2.655 & 0.1854 \\
\hline $65(14)$ & 0 & $36(2.88 \%)$ & 0 & 0.4086 \\
\hline 70 & 0 & $1(0.08 \%)$ & 0 & 0.4086 \\
\hline 73 & 0 & $1(0.08 \%)$ & 0 & 0.4086 \\
\hline $75(15)$ & 0 & $1(0.08 \%)$ & 0 & 0.4086 \\
\hline
\end{tabular}


Table 1c. HLA - DRB1 alleles and CMV disease in kidney transplantation patients

\begin{tabular}{ccccc}
\hline Allele & $\begin{array}{c}\text { Alleles from patients with } \\
\text { CMV disease } \\
\mathbf{7 0}\end{array}$ & $\begin{array}{c}\text { Alleles from patients with- } \\
\text { out CMV disease } \\
\mathbf{1 2 7 8}\end{array}$ & OR & p value \\
\hline$* 1$ & $3(4.28 \%)$ & $117(9.15 \%)$ & 0.44 & 0.2885 \\
\hline$* 03$ & $5(7.14 \%)$ & $174(13.61 \%)$ & 0.49 & 0.3494 \\
\hline$* 4$ & $5(7.14 \%)$ & $128(10.01 \%)$ & 0.69 & 0.6106 \\
\hline$* 07$ & $7(10 \%)$ & $104(8.13 \%)$ & 1.25 & 0.5181 \\
\hline$* 08$ & $1(1.42 \%)$ & $21(1.64 \%)$ & 0.87 & 0.8787 \\
\hline$* \mathbf{0 9}$ & $\mathbf{0}$ & $\mathbf{8 ( 0 . 6 2 \% )}$ & $\mathbf{0}$ & $\mathbf{0 . 0 4 1 9}$ \\
\hline$* 10$ & $1(1.42 \%)$ & $14(1.09 \%)$ & 1.31 & 0.4205 \\
\hline$* 11$ & $19(27.14 \%)$ & $293(22.92 \%)$ & 1.25 & 0.826 \\
\hline$* 12$ & $1(1.42 \%)$ & $19(1.48 \%)$ & 0.96 & 0.9561 \\
\hline$* 13$ & $7(10 \%)$ & $113(8.84 \%)$ & 1.14 & 0.6641 \\
\hline$* 14$ & $4(5.71 \%)$ & $72(5.63 \%)$ & 1.01 & 0.8609 \\
\hline$* 15$ & $8(11.42 \%)$ & $99(7.74 \%)$ & 1.58 & 0.2488 \\
\hline$* 16$ & $9(12.85 \%)$ & $116(9.07 \%)$ & 1.49 & 0.3001 \\
\hline
\end{tabular}

CMV disease. The difference in HLA frequencies between these two groups was statistically significant. Association analysis of HLA-A reveals the rare A36 allele that was excluded. HLA-B with greater allelic polymorphism has two alleles predispose to CMV disease: B48 and B57(Table 1b). Concerning HLA-DRB1, our results reveals one protective allele against developing CMV disease: DRB1*09 and no alleles for this locus which give susceptibility to CMV disease (Table 1c). We focused our study on patients CMV disease after kidney transplantation. Many investigators showed the importance of the HLA system in the anti-viral responses, especially against the CMV pathogen. Several reports showed that HLA-A2 and HLA-DR11 increase and HLA-B16 reduces the risk for CMV infection [12]. The other studies have shown that HLA-DR7 have increased risk for CMV infection [13], while Retierre et al. [14] reported a different HLA class I gene A11, A32 and HLA class II gene DR11 with prevalence in the viral infection among solid organ recipients. However, other investigators found that HLA-A11 increases the risk for CMV infection in kidney graft re- cipients [15]. Kekik et al. have demonstrated a higher incidence of HLA-A30, HLA-B40, and HLA-DRB1*15 CMV infection [16]. We found that HLA-B48 and HLA-B57 have a significant influence in CMV disease after transplantation and HLA-DRB1*09 indicates the opposite results. In the literature, HLA alleles are recognized as risk factors for CMV disease, but some of them can have a protective role [16-19], and this situation might be useful pretransplant in estimating the risk of CMV disease after transplantation and designing individualized therapy. A better understanding of the different HLA associated immune mechanism within CMV disease may lead to improved management strategies in kidney transplantation. The identification of the genes which are involved in the pathogenesis of an infectious disease is very important for the development of new therapeutic strategies or even new therapies for that infectious disease.

Simona Luscalov ${ }^{1}$, Dan Adrian Luscalov ${ }^{2 *}$, Luminita Ioana Loga ${ }^{2}$, Adriana Milena Muntean ${ }^{2}$, Gabriel Cristian Dragomir Loga ${ }^{3}$, Lucia Dican ${ }^{1}$ 
1. "Iuliu Hatieganu" University of Medicine and Pharmacy, Faculty of Medicine Cluj Napoca, Romania

2. Clinical Institute of Urology and Renal

Transplantation Cluj, National Transplant

Agency

3. Technical University, Faculty of Automation and Computer Science Cluj Napoca, Romania

\section{Corresponding author}

Luscalov Dan Adrian, e-mail: aluscalov@ yahoo.com

Received: $30^{\text {th }}$ August 2016

Accepted: 22 $2^{\text {nd }}$ October 2017

Published: 23 ${ }^{\text {rd }}$ October 2017

\section{Conflicts of interest}

The authors declare no conflicts of interest.

\section{Acknowledgements}

This paper was published under the frame of European Social Found, Human Resources Development Operational Programme 2007-2013 project no. POSDRU/159/1.5/138776

\section{References}

1. Brennan D. Cytomegalovirus in Renal Transplantation. J Am Soc Nephrol. 2011;12: 848-55.

2. Bowden RA, Ljungman P, Snydman DR. Transplant Infections. Lippincott Williams \& Wilkins. 2010;23:328-9.

3. Luscalov S, Loga LI, Dican L, Junie LM. Cytomegalovirus infection in Immunosuppressed infection patients after kidney transplantation. Clujul Medical. Ahead of print 2016. DOI: $10.15386 /$ cjmed-587

4. Eid AJ, Razonable RR. Cytomegalovirus disease in solid organ transplant recipients: advances lead to new challenges and opportunities. Curr Opin Organ Transplant. 2007;12:610-7. DOI: 10.1097/ MOT.0b013e3282f0d386

5. Stratta RJ. Clinical patterns and treatment of cytomegalovirus infection after solid organ transplantation. Transplant Proc. 1993;25:15-21.

6. Emery VC, Hassan-Walker AF, Burroughs A, Griffiths PD. Human cytomegalovirus (CMV) replication dynamics in CMV-native and experienced immunocompromised hosts. J Infect Dis. 2002;185:1723-8. DOI: 10.1086/340653

7. Chou SW. Reactivation and recombination of multiple cytomegalovirus strains from individual organ donors. J Infect Dis. 1989;160:11-5. DOI: 10.1093/ infdis/160.1.11

8. Chou SW. Acquisition of donor strains of cytomegalovirus by renal-transplant recipients. $\mathrm{N}$ Engl J Med. 1986;314:1418-23. DOI: 10.1056/ NEJM198605293142205

9. Rubin RH. Infectious disease complications of renal transplantation. Kidney Int. 1993; 44:221. DOI: 10.1038/ki.1993.234

10. Terasaki PI. The history of HLA and Transplantation. Hirosaki Med J. 2013;64. Suppl. S45-52.

11. Dickenmann MJ, Cathomas G, Steiger J, Mihatsch MJ, Thiel G, Tamm M. Cytomegalovirus infection and graft rejection in renal transplantation. Transplantation 2001;71:764-7. DOI: 10.1097/00007890-20010327000013

12. Fan J, Meng XQ, Yang MF, Zhou L, Chen XM, Hu MJ, Fan WW, Ma WH, Li LJ. Association of cytomegalovirus infection with human leukocyte antigen genotypes in recipients after allogeneic liver transplantation. Hepatobiliary Pancreat Dis Int. 2006 Feb;5(1):34-8.

13. Kraat YJ, Christiaans MH, Nieman FH, van den BergLoonen PM, van Hooff JP, Bruggeman CA. Increased frequency of CMV infection in HLA-DR7 matched renal allograft recipients. Lancet. 1993;341(8843):4945. DOI: 10.1016/0140-6736(93)90248-F

14. Retiere C, Lesimple B, Lepelletier D, Bignon JD, Hallet MM, Imbert-Marcille BM. Association of glycoprotein B and immediate early-1 genotypes with human leukocyte antigen alleles in renal transplant recipients with cytomegalovirus infection. Transplantation. 2003;75(1):161-5. DOI: 10.1097/00007890200301150-00030 
15. Filipov J, Paskalev E, Simeonov P, Mihaylova A, Naumova E. HLA-A11 Increases the Risk for Post-Transplant Active Cytomegalovirus Infection in Kidney Graft Recipients. BANTAO Journal. 2010;8(1):44-9.

16. Kekik C, Besisik BK, Seyhun Y, Oguz FS, Sargin D, Carin MN. Relationship Between HLA Tissue Type, CMV Infection, and Acute Graft-vs-Host Disease After Allogeneic Hematopoietic Stem Cell Transplantation: Single-Center Experience. Transplant Proc. 2009; 41(9):3515-970. DOI: 10.1016/j.transproceed.2009.04.017

17. Bal Z, Uyar ME, Tutal E, Erdogan E, Colak T, Sezer $\mathrm{S}$, et al. Cytomegalovirus infection in renal trans- plant recipients: one center's experience. Transplant Proc. 2013;45(10):3520-3. DOI: 10.1016/j.transproceed.2013.08.098

18. Varga M, Rajczy K, Telkes G, Hidvegi M, Peter A, Remport A, et al. HLA-DQ3 is a probable risk factor for CMV infection in high-risk kidney transplant patients. Nephrol Dial Transplant. 2008;23(8):2673-8. DOI: $10.1093 /$ ndt/gfn111

19. Futohi F, Saber A, Nemati E, Einollahi B, Rostami Z. Human Leukocyte Antigen Alleles and Cytomegalovirus Infection After Renal Transplantation. Nephro Urol Mon. 2015;7(6):1-5. DOI: 10.5812/numonthly. 31635 